\title{
MicroRNA-543 suppresses liver cancer growth and induces apoptosis via the JAK2/STAT3 signaling pathway
}

\author{
DIANHUI XIU ${ }^{1 *}$, DAWEI WANG ${ }^{2 *}$, JING WANG $^{3}$, FUJIAN JI $^{4}$ and WENLEI ZHANG ${ }^{5}$ \\ ${ }^{1}$ Department of Radiology, The China Japan Union Hospital of Jilin University; \\ Departments of ${ }^{2}$ Emergency and ${ }^{3}$ Gastrointestinal Medicine, First Hospital of Jilin University; \\ ${ }^{4}$ Department of General Surgery, The China Japan Union Hospital of Jilin University; \\ ${ }^{5}$ Department of Interventional Therapy, First Hospital of Jilin University, Changchun, Jilin 130021, P.R. China
}

Received June 14, 2016; Accepted September 6, 2018

DOI: $10.3892 / \mathrm{ol} .2018 .9838$

\begin{abstract}
The aim of the present study was to investigate the function of microRNA-543 on liver cancer cell proliferation and apoptosis, and also to identify whether the Janus kinase 2 (JAK2)/signal transducer and activator of transcription 3 (STAT3) signaling pathway was a direct target of microRNA-543. When compared with paracarcinoma tissue, microRNA-543 expression in tissue samples from patients with liver cancer was identified to be decreased. Furthermore, overall survival of patients with high microRNA-543 expression was increased compared with patients with low microRNA-543 expression. Inhibition of microRNA-543 expression increased cell proliferation and decreased apoptosis of liver cancer cells. It also activated the protein expression of phosphorylated JAK2, phosphorylated STAT3, c-Myc and B-cell lymphoma 2 (Bcl-2) in liver cancer cells. However, activation of microRNA-543 expression in turn decreased cell proliferation and increased apoptosis of liver cancer cells. The results of the present study highlight the pivotal function of microRNA-543 as an inhibitor in liver cancer cell proliferation by observing JAK2/STAT3/c-Myc/Bcl-2 in liver cancer.
\end{abstract}

\section{Introduction}

Liver cancer is a common type of gastrointestinal cancer, whose incidence is only second to that of stomach cancer (1).

Correspondence to: Mr. Fujian Ji, Department of General Surgery, The China Japan Union Hospital of Jilin University, 126 Xiantai Street, Changchun, Jilin 130021, P.R. China

E-mail: abbsdan@126.com

Mr. Wenlei Zhang, Department of Interventional Therapy, First Hospital of Jilin University, 71 Xinmin Street, Changchun, Jilin 130021, P.R. China

E-mail: zbnxdzv381@126.com

${ }^{*}$ Contributed equally

Key words: microRNA-543, liver cancer, Janus kinase 2, signal transducer and activator of transcription 3, c-Myc, B-cell lymphoma 2
In China, there are numerous patients with hepatitis, among whom hepatitis B is more common, and hepatitis B is often transferred into hepatitis B-cirrhosis-liver cancer (1). However, the clinical treatment of liver cancer is not ideal, which primarily results from the recurrence and metastasis of hepatocellular carcinoma (HCC) $(1,2)$. Surgery remains the most effective treatment of liver cancer, but surgical resection is aimed only at the visible tumor, and it cannot remove the invisible small tumor foci or micro-metastases. Therefore, seeking an effective treatment method to inhibit tumor recurrence has become a hot research topic.

The Janus kinase (JAK)/signal transducer and activator of transcription (STAT) signaling pathway overactivated in a number of tumors is a common signal transduction pathway in tumors (3). It is has been identified that the excessive activation of STAT is associated with the recurrence and metastasis of various tumors, including breast cancer, lung cancer, colon cancer, stomach cancer, glioma, melanoma and other skin squamous cell carcinomas (4). The JAK/STAT signaling pathway is able to regulate cell proliferation, apoptosis and angiogenesis (5). STAT has been identified to be excessively expressed in HCC tissues, and it is positively correlated with the clinical staging and grading of $\mathrm{HCC}$ (5).

It has been identified recently that the expression levels of microRNAs in various tumors are abnormal to various degrees, and they change upward or downward, which indicates microRNAs have a certain association with the development of tumors (5). The association between microRNAs and cancer is an important focus of research worldwide and has led to an increasing number of novel results and hypotheses (6). The aim of the present study was to investigate the function of microRNA-543 on liver cancer cell proliferation and apoptosis, and also to identify whether the JAK2/STAT3 signaling pathway was a direct target of microRNA-543.

\section{Materials and methods}

Ethics statement and samples. The present study was approved by the institutional ethics and scientific committee of The China Japan Union Hospital of Jilin University (Changchun, China). All patients provided written informed consent for the use of their tissues. All HCC tumor tissues and matched 

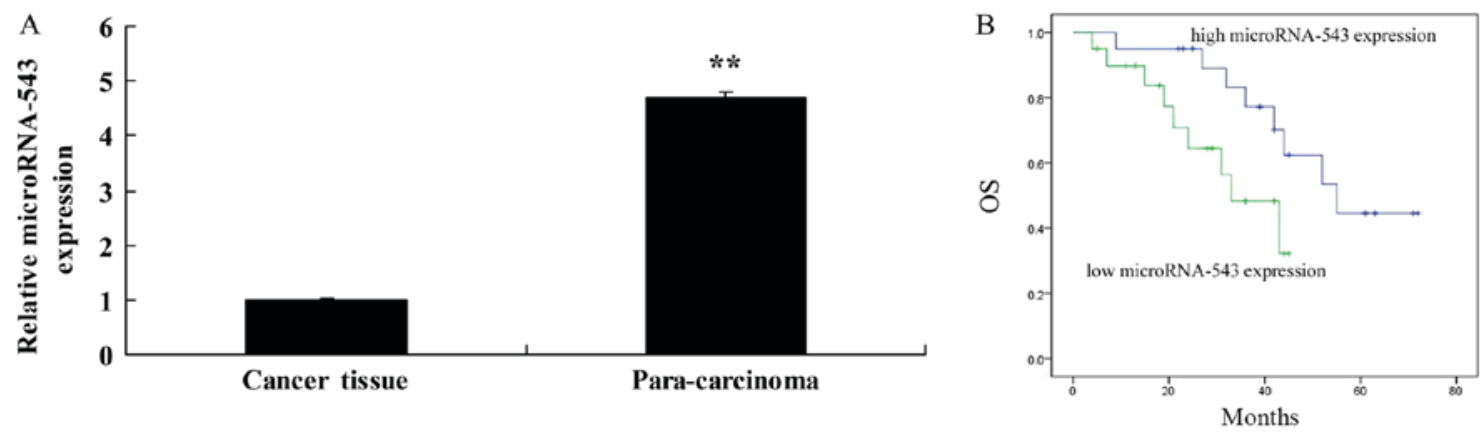

Figure 1. MicroRNA-543 expression and OS. (A) MicroRNA-543 expression. (B) Overall survival. OS, overall survival. ${ }^{* *}$ P $<0.01$ vs. cancer tissue group.

adjacent normal tissues $(\mathrm{n}=56$; male; between 57 and 79 years of age) were collected at the Department of Gastrointestinal Medicine, The China Japan Union Hospital of Jilin University between March 2015 and May 2015. Tissue samples were collected and stored at $-80^{\circ} \mathrm{C}$ until further use.

Reverse transcription-quantitative polymerase chain reaction ( $R T-q P C R)$. Total RNA was prepared from tissues samples using TRIzol ${ }^{\circledR}$ reagent (Invitrogen; Thermo Fisher Scientific, Inc., Waltham, MA, USA), according to the manufacturer's protocol. A $100 \mathrm{ng}$ amount of total RNA was reverse-transcribed into cDNA using a TaqMan ${ }^{\mathrm{TM}}$ MicroRNA Reverse Transcription kit (Applied Biosystems; Thermo Fisher Scientific, Inc.), according to the manufacturer's protocol. Subsequently, qPCR was performed using SYBR Select Master mix for CFX (Invitrogen; Thermo Fisher Scientific, Inc.) to quantify microRNA-543 expression. microRNA-543 forward, 5'-TGGCAAAGGAGCAGATTAGTAGG-3', and reverse, 5'-CTGCCACAAGCCACTAGAGGATAAGA-3'; U6 forward, 5'-GCTTCGGCAGCACATATACTAAAA T-3' and reverse, 5'-CGCTTCACGAATTTGCGTGTCAT-3'. microRNA expression was measured using the comparative cycle quantification $\left(\mathrm{C}_{q} ; 2^{-\Delta \Delta C \mathrm{C}}\right)$ method (7).

Cell culture. HCC MHCC97 and Hep3B cells were obtained from the Experiment Center of Jilin University and maintained in RPMI-1640 medium (Gibco; Thermo Fisher Scientific, Inc.) supplemented with $10 \%$ fetal bovine serum (Hyclone; GE Healthcare Life Sciences, Logan, UT, USA) at $37^{\circ} \mathrm{C}$ in a humidified atmosphere containing $5 \% \mathrm{CO}_{2}$.

Cell transfection. Negative control (5'-CCCCCCCCCCCC CC-3'), microRNA-543 inhibitor (5'-ATATATGCTCTGGAA ATGATTCAGCAGTACGAAAGCC-3') and microRNA-543 activator (5'-GGCTTTCGTACTGCTGAATCATTTCCAGAG CATATAT-3') were synthesized by Shanghai GenePharma Co., Ltd. (Shanghai, China). Plasmids were transfected into MHCC97 and Hep3B cells using Lipofectamine ${ }^{\circledR} 2000$ reagent (Invitrogen; Thermo Fisher Scientific, Inc.) to a final concentration of $20 \mathrm{nmol} / \mathrm{l}$.

Cell proliferation assay. MHCC97 and Hep3B cells transfection were seeded on 96-well plates at an initial density of $3 \times 10^{3}$ cells/well. A $100 \mu \mathrm{l}$ volume of sterile MTT $(0.5 \mathrm{mg} / \mathrm{ml}$; Sigma-Aldrich; Merck KGaA, Darmstadt, Germany) was added to the cells for $4 \mathrm{~h}$ following 0,24 and $48 \mathrm{~h}$ of incubation at $37^{\circ} \mathrm{C}$.
The culture medium was removed and $150 \mu 1$ dimethylsulfoxide was added for $30 \mathrm{~min}$ to dissolve the formazan product. Optical density was determined at $490 \mathrm{~nm}$.

Western blot analysis. Transfected MHCC97 and Hep3B cells were seeded on 6-well plates at initial density of $1 \times 10^{6}$ cells/well. Proteins were extracted using radioimmunoprecipitation lysis buffer (Thermo Fisher Scientific, Inc.) and the protein content was determined using a Bicinchoninic Acid Protein assay kit (Beyotime Institute of Biotechnology, Haimen, China). Equal amounts of proteins $(50 \mu \mathrm{g})$ were subjected to $10 \%$ SDS-PAGE and then transferred onto polyvinylidene difluoride membranes. Following blocking with 5\% non-fat milk Tris-buffered saline containing $0.1 \%$ Tween- 20 (TBST) for $1 \mathrm{~h}$ at $37^{\circ} \mathrm{C}$, the membranes were washed with TBST for $1 \mathrm{~h}$ at room temperature, and incubated with antibodies against phospho- (p-)JAK2 (66245; 1:1,000), p-STAT3 (52075; 1:1,000), c-Myc (9402; 1:1,000), and B-cell lymphoma 2 (Bcl-2; 15071; 1:1,000) and GAPDH (5174; 1:5,000; all from Cell Signaling Technology, Inc., Danvers, MA, USA) and GAPDH overnight at $4^{\circ} \mathrm{C}$. The membrane was washed with TBST and incubated with a horseradish peroxidase-conjugated anti-rabbit IgG antibody (7074; 1:5,000; Cell Signaling Technology, Inc.) for $1 \mathrm{~h}$ at $37^{\circ} \mathrm{C}$. The membrane was detected with BeyoECL Star (Beyotime Institute of Biotechnology) reagent and images were captured using Image Lab 3.0 (Bio-Rad Laboratories, Inc., Hercules, CA, USA).

Statistical analysis. All quantitative data are expressed as the mean \pm standard deviation. Kaplan-Meier survival analysis and log-rank test were used to assess overall survival (OS) rates. Statistical evaluation was performed using Student's t-test. $\mathrm{P}<0.05$ was considered to indicate a statistically significant difference.

\section{Results}

MicroRNA-543 expression and OS. Using RT-qPCR, it was identified that microRNA-543 levels were significantly decreased in the HCC tissues in comparison with the adjacent tissues (Fig. 1A). It was also identified that OS rates of patients with high microRNA-543 expression were increased, compared with that of patients with low microRNA-543 expression (Fig. 1B).

Inhibition of microRNA-543 expression increases cell proliferation and inhibits cell apoptosis of liver cancer cells. 

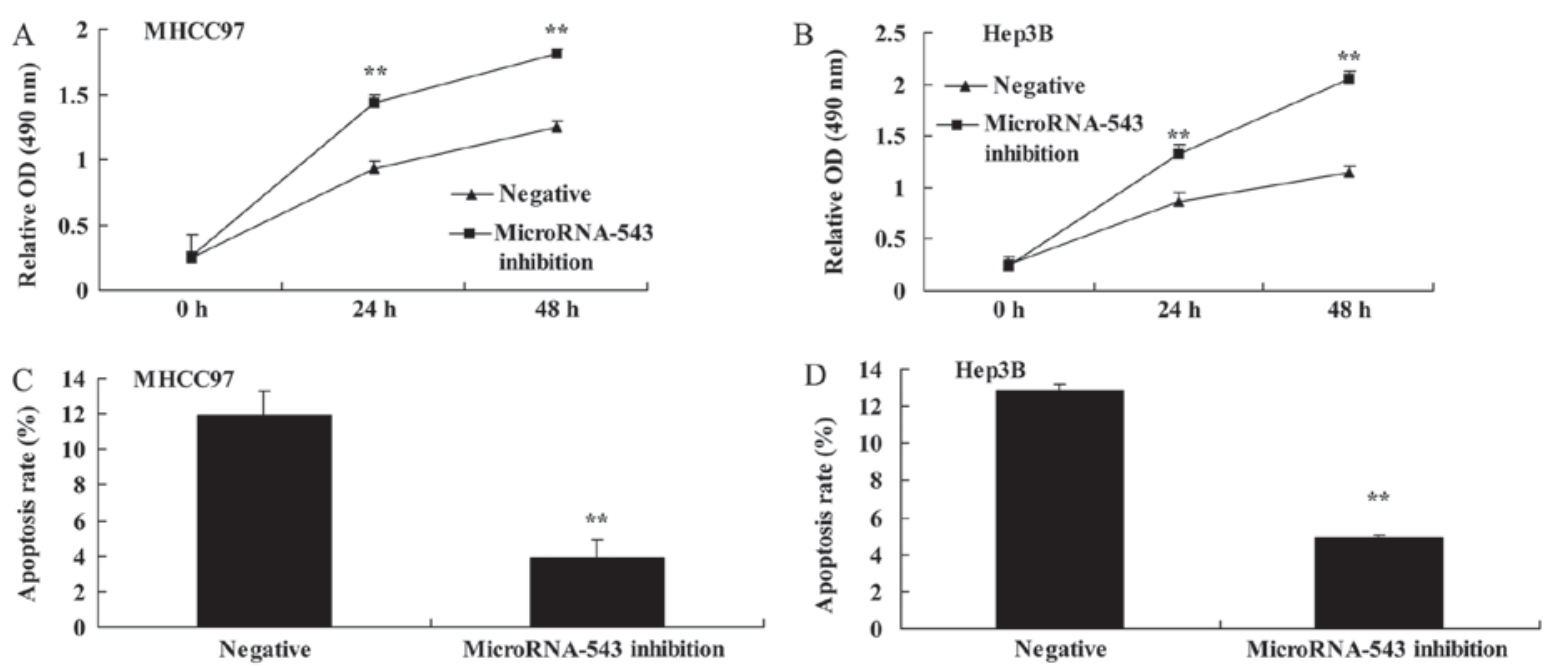

Figure 2. Inhibition of microRNA-543 expression increases proliferation and inhibits apoptosis of liver cancer cells. Inhibition of microRNA-543 expression increased proliferation of (A) MHCC97 cells and (B) Hep3B cells, and inhibited apoptosis of (C) MHCC97 cells and (D) Hep3B cells. ${ }^{* *}$ P $<0.01$ vs. negative group. OD, optical density.

A

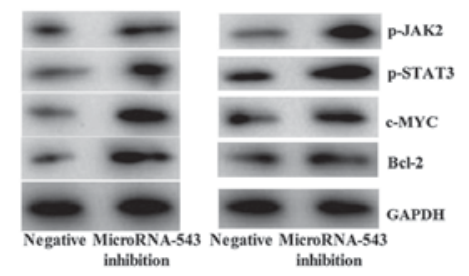

$\mathrm{D}$

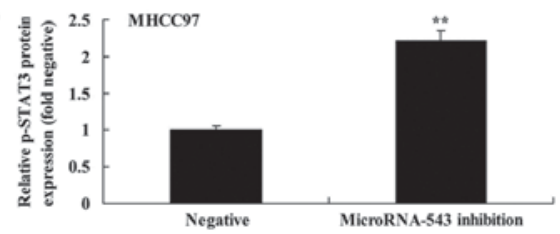

G

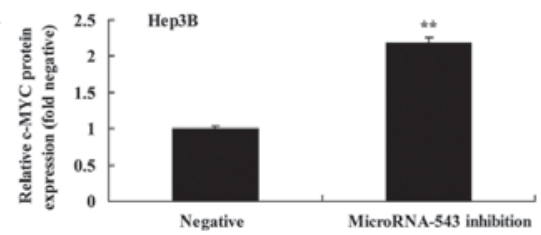

B
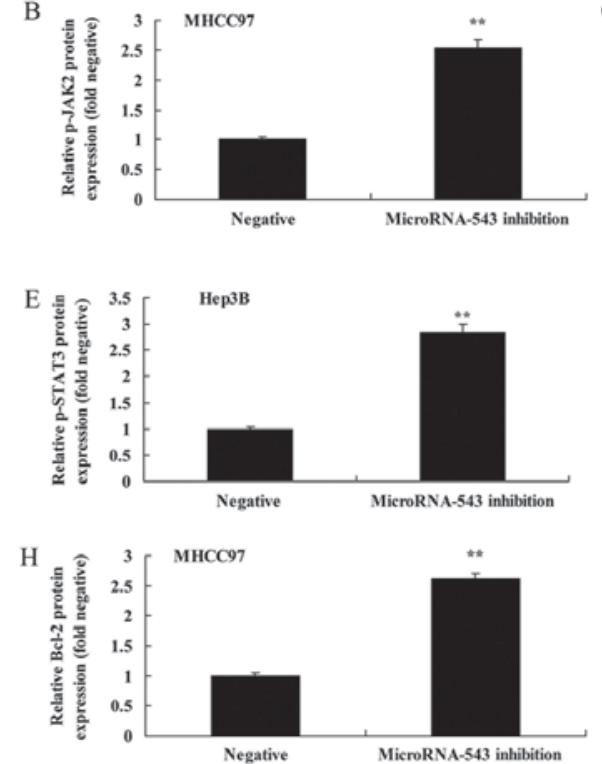
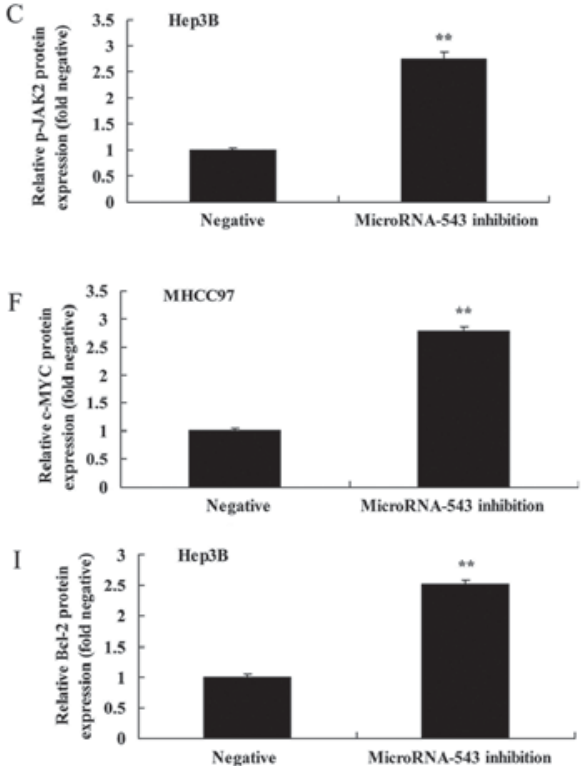

Figure 3. Inhibition of microRNA-543 expression increases the protein expression of p-JAK2, p-STAT3, c-Myc and Bcl-2 in liver cancer cells. (A) Western blot analysis of p-JAK2, p-STAT3, c-Myc and Bcl-2 proteins in (left) MHCC97 and (right) Hep3B cells. Quantification of p-JAK2 in (B) MHCC97 and (C) Hep3B cells, p-STAT3 in (D) MHCC97 and (E) Hep3B cells, c-Myc in (F) MHCC97 and (G) Hep3B cells, and Bcl-2 in (H) MHCC97 and (I) Hep3B cells. ${ }^{* *} \mathrm{P}<0.01$ vs. negative group. p-, phospho-; JAK2, Janus kinase 2; STAT3, signal transducer and activator of transcription 3; Bcl-2, B-cell lymphoma 2.

To investigate whether the inhibition of microRNA-543 expression had an effect on HCC cell proliferation and apoptosis, MHCC97 and Hep3B HCC cells were transfected with microRNA-543 inhibitor. The proliferation of MHCC97 and Hep3B cells transfected with microRNA-543 inhibitor was significantly increased compared with that of the negative control group (Fig. 2A and B). The apoptosis rate of MHCC97 and Hep3B cells transfected with microRNA-543 inhibitor was significantly decreased compared with that of the negative control group (Fig. 2C and D).

Inhibition of microRNA-543 expression activates the protein expression of $p$-JAK2, p-STAT3, $c-M y c$ and Bcl-2 in liver cancer cells. The effect of inhibiting microRNA-543 on the expression of various proteins in HCC cells was investigated. As presented in Fig. 3, the inhibition of microRNA-543 expression significantly increased p-JAK2, p-STAT3, c-Myc and $\mathrm{Bcl}-2$ protein expression in MHCC97 and Hep3B cells, compared with the negative control group.

Activation of microRNA-543 expression decreases cell proliferation and induces cell apoptosis of liver cancer cell. To further investigate the function of microRNA-543 in HCC progression, the expression of microRNA-543 was activated in MHCC97 and Hep3B cells. Activation of microRNA-543 expression significantly decreased cell proliferation (Fig. 4A and B) and induced apoptosis (Fig. 4C and D) of MHCC97 and Hep3B cells, compared with the negative control group. 

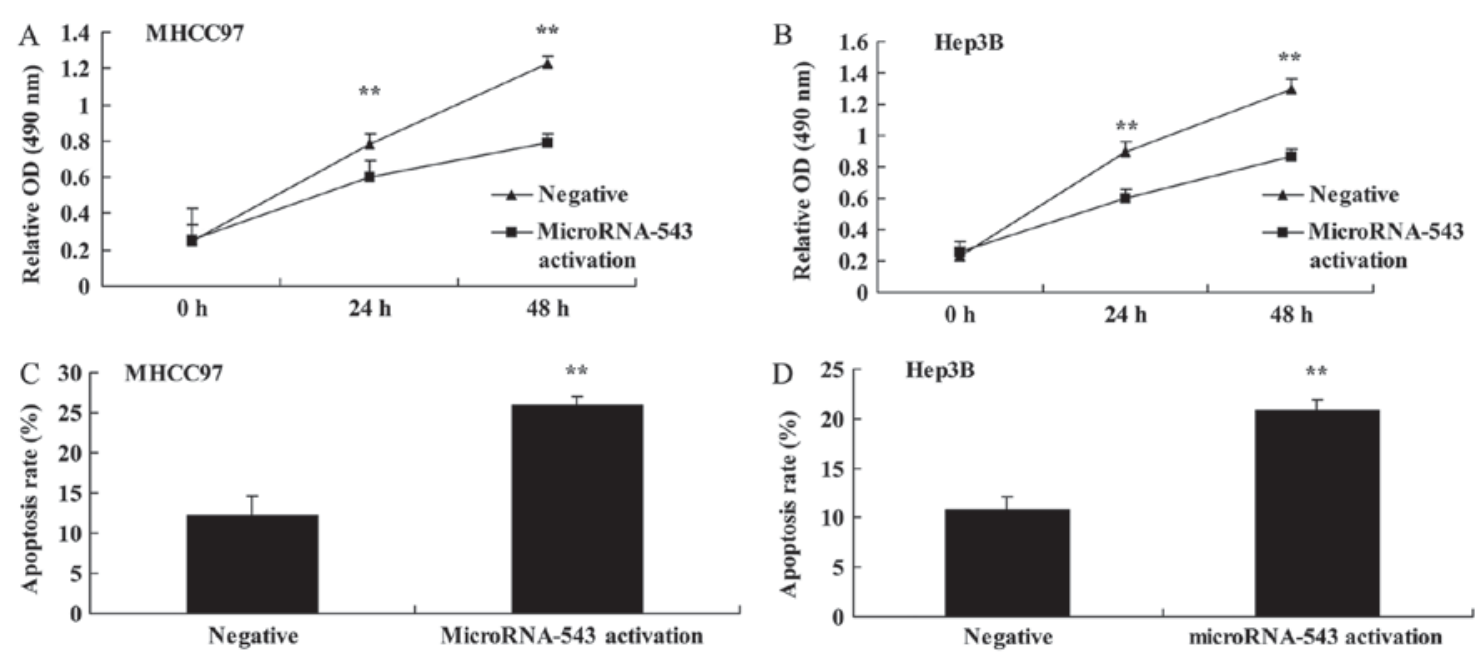

Figure 4. Activation of microRNA-543 expression decreases proliferation and induces apoptosis of liver cancer cells. Activation of microRNA-543 expression decreased the proliferation of (A) MHCC97 and (B) Hep3B cells, and increased apoptosis of (C) MHCC97 and (D) Hep3B cells. ${ }^{* * *} \mathrm{P}<0.01$ vs. negative group.

A
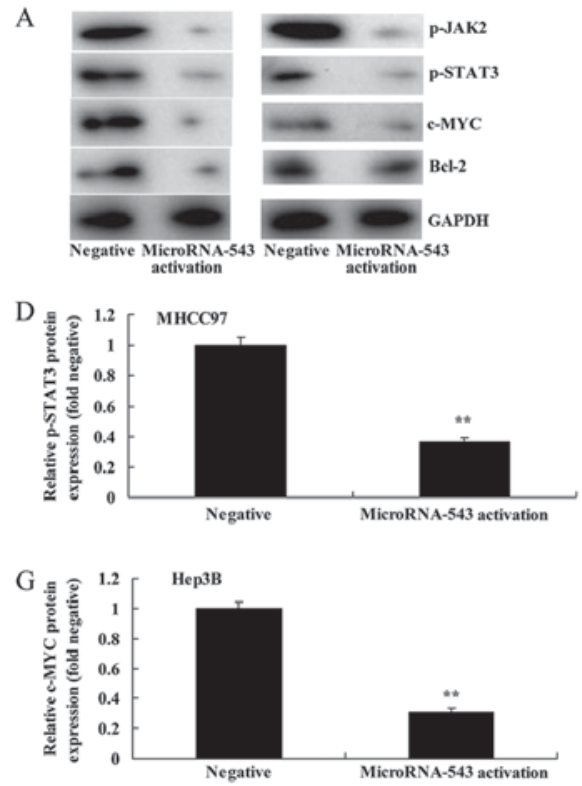
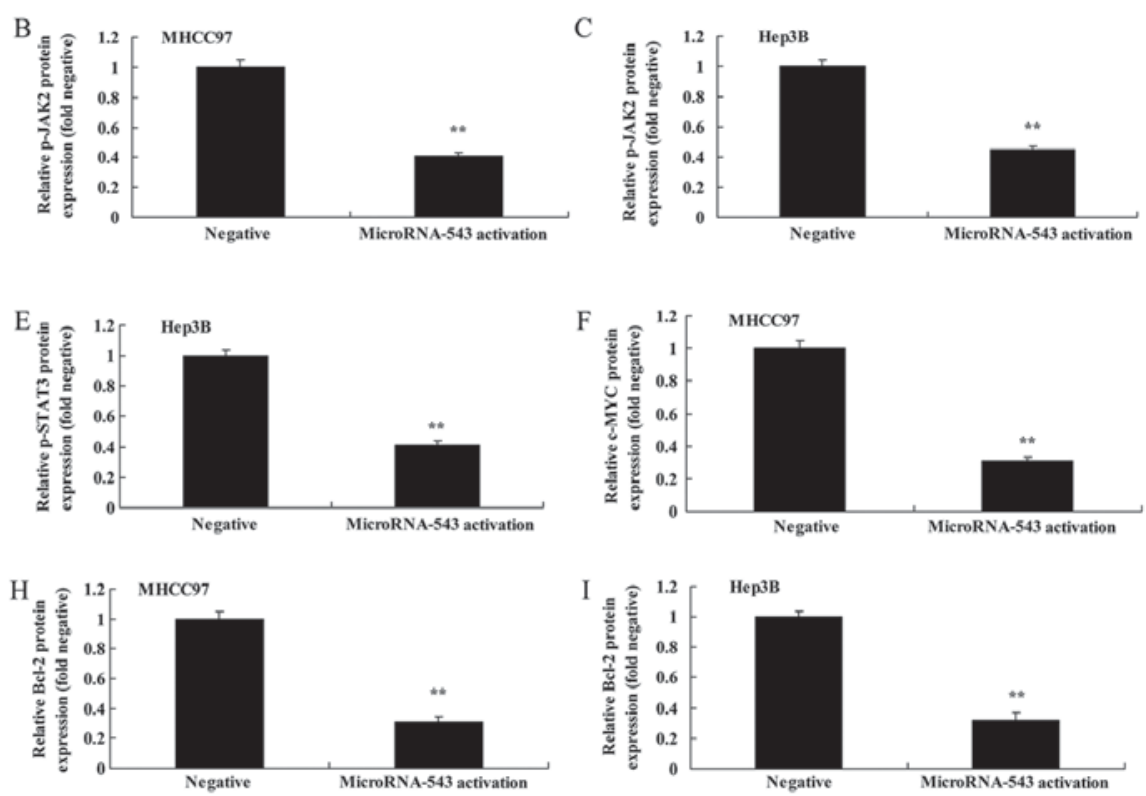

Figure 5. Activation of microRNA-543 expression decreases the protein expression of p-JAK2, p-STAT3, c-Myc and Bcl-2 in liver cancer cells. (A) Western blot analysis of p-JAK2, p-STAT3, c-Myc and Bcl-2 proteins in (left) MHCC97 and (right) Hep3B cells. Quantification of p-JAK2 in (B) MHCC97 and (C) Hep3B cells, p-STAT3 in (D) MHCC97 and (E) Hep3B cells, c-Myc in (F) MHCC97 and (G) Hep3B cells, and Bcl-2 in (H) MHCC97 and (I) Hep3B cells. ${ }^{* *} \mathrm{P}<0.01$ vs. negative group. p-, phospho-; JAK2, Janus kinase 2; STAT3, signal transducer and activator of transcription 3; Bcl-2, B-cell lymphoma 2.

Activation of microRNA-543 expression decreases the protein expression of $p$-JAK2, $p$-STAT3, $c$-Myc and Bcl-2 in liver cancer cells. Western blot analysis was used to analyze the protein expression of p-JAK2, p-STAT3, c-Myc and Bcl-2 protein expression in MHCC97 and Hep3B cells transfected with microRNA-543 activator. It was identified that p-JAK2, p-STAT3, c-Myc and Bcl-2 protein expression in MHCC97 and Hep3B cells transfected with microRNA-543 activator was significantly inhibited, compared with the negative control group (Fig. 5).

\section{Discussion}

Liver cancer is the most common malignant liver disease in adults (8). Although the success of its therapy is improving continuously, and there are a variety of therapies, including surgical resection, liver transplantation, radiotherapy and chemotherapy, none has significantly extended the mean 5-year survival of patients with liver cancer $(9,10)$. One of the most important reasons for the high mortality and poor prognosis of patients with liver cancer is that the underlying molecular mechanism is poorly understood. However, with the development of modern cell molecular biology, it has been identified that the formation of liver cancer is typically accompanied by a series of abnormal molecules and signaling pathways. Therefore, investigating the underlying molecular mechanism and treat the characteristics of molecules as antitumor targets may open up a new avenue for the clinical prevention and treatment of liver cancer (8). The results of the present study indicated that the activation of microRNA-543 expression decreases proliferation 
and induces apoptosis of liver cancer cells, which suggests that microRNA-543 may function as a tumor suppressor in HCC.

The JAK/STAT signaling pathway is a well-known signal transduction pathway, which is typically overexpressed in a number of tumors (11). It has been reported that excessive activation of STAT is markedly associated with the invasion and metastasis of breast cancer, lung cancer, colon cancer, stomach cancer, glioma, melanoma, squamous cell carcinoma and other tumors (12). The JAK/STAT signaling pathway may affect, directly or indirectly, cell proliferation, apoptosis, invasion, metastasis and angiogenesis. Furthermore, overexpression of STAT has been identified in HCC, which is positively correlated with the clinical staging and pathological grading of HCC (13). In the JAK/STAT signaling pathway, the interaction of external stimulatory factors and cell membranes leads to receptor dimerization, which can activate JAK molecules in the cytoplasm (14). The activated JAK is coupled with cytokine receptors on the cell membrane, which phosphorylates the tyrosine residues of the receptor, and the phosphorylation site becomes the recognition site of the Src homology 2 domain in STAT proteins. Subsequently, STAT binds to the receptor and is phosphorylated by JAK (15). When the affinity between phosphorylated STAT and their receptors decreases, the phosphorylated STAT separates from its receptor and generates the active form of dimer. It is subsequently transferred to the cell nucleus and binds to specific DNA-response elements to result in the expression of corresponding genes [e.g., vascular endothelial growth factor (VEGF) and matrix metalloproteinases (MMPs)], the synthesis of a variety of proteins (e.g., VEGF and MMPs), and the performance of various biological behaviors (15). The results of the present study indicated that the activation of microRNA-543 limits the protein expression of p-JAK2 and p-STAT3 in MHCC97 and Hep3B cells, thus indicating that microRNA-543 regulates HCC cell proliferation through the JAK2/STAT3 signaling pathway.

The cell cycle is a series of ordered cellular activities which cause the division and duplication of its DNA to create two daughter cells (16). The entire process is divided into two periods: Silence period and proliferative period. However, by contrast, cancer is disorderly cell proliferation (17). The cell cycle is associated with the regulation of a number of proteins. In the cell transition phase from $G_{2}$ to $M$, the mitosis-promoting factor is the most important regulatory element (17). c-Myc distribution results in different types of liver cancer tissue, which means that there are distinct differences between poorly differentiated adenocarcinoma and highly or moderately differentiated adenocarcinoma, and undifferentiated adenocarcinoma is different from differentiated adenocarcinoma. Consequently, it can be concluded that positive c-Myc expression is associated with the degree of tumor differentiation.c-Myc is slightly increased in slow-growing and highly differentiated adenocarcinoma, whereas it is significantly increased in fast-growing and poorly differentiated adenocarcinoma $(16,18)$. In addition, in the present study, it was identified that microRNA-543 activation represses c-Myc expression of MHCC97 and Hep3B cells. Thus, c-Myc expression may be a crucial signaling pathway through which microRNA-543 affects HCC.
Tumorigenesis arises owing to the imbalance between cell proliferation and apoptosis. It has been identified that there is abnormal expression of various cancer genes, tumor suppressor genes and apoptosis genes in HCC. Among them, Bcl-2 and c-Myc genes are key representatives (19). The proto-oncogene $\mathrm{Bcl}-2$ was originally identified in the chromosomal translocation point of B-cell lymphoma. The proto-oncogene $\mathrm{Bcl}-2$, as an anti-apoptotic gene, is predicated to be associated with tumor resistance and anti-radiation sensitivity (20). Previous studies have indicated that Bcl-2 and its protein product are expressed in benign and malignant tissue of several non-hematopoietic systems, except lymphoma, such as lung cancer, stomach cancer and breast cancer; and they affect the occurrence and development of tumors $(19,21)$. Transgenic animal experiments revealed that Bcl-2 expression by liver cells may be unaffected by Fas-mediated hepatocyte apoptosis, which leads to tumorigenesis (21). It was identified that microRNA-543 activation also significantly decreases $\mathrm{Bcl}-2$ protein expression in MHCC97 and Hep3B cells.

In conclusion, the results of the present study indicated that microRNA-543 inhibits liver cancer growth and leads to apoptosis via the JAK2/STAT3 signaling pathway. The results also suggested that microRNA-543 may serve as a novel diagnostic and prognostic biomarker for HCC.

\section{Acknowledgements}

Not applicable.

\section{Funding}

The present study was supported by the Natural Science Foundation of China (grant no. 81571939).

\section{Availability of data and materials}

The datasets used and/or analyzed during the current study are available from the corresponding author on reasonable request.

\section{Authors' contributions}

FJ designed the experiment. DX, DW, JW and WZ performed the experiment. FJ and DX analyzed the data. FJ wrote the manuscript.

\section{Ethics approval and consent to participate}

The present study was approved by the institutional ethics and scientific committee of The China Japan Union Hospital of Jilin University. All patients provided written informed consent for the use of their tissues.

\section{Patient consent for publication}

Not applicable.

\section{Competing interests}

The authors declare that they have no competing interests. 


\section{References}

1. Rauf N, Tahir SS, Dilawar S, Ahmad I and Parvez S: Serum selenium concentration in liver cirrhotic patients suffering from hepatitis B and C in Pakistan. Biol Trace Elem Res 145: 144-150, 2012.

2. Gabrielson A, Tesfaye AA, Marshall JL, Pishvaian MJ, Smaglo B, Jha R, Dorsch-Vogel K, Wang H and He AR: Phase II study of temozolomide and veliparib combination therapy for sorafenib-refractory advanced hepatocellular carcinoma. Cancer Chemother Pharmacol 76: 1073-1079, 2015.

3. Senggunprai L, Kukongviriyapan V, Prawan A and Kukongviriyapan U: Quercetin and EGCG exhibit chemopreventive effects in cholangiocarcinoma cells via suppression of JAK/STAT signaling pathway. Phytother Res 28: 841-848, 2014.

4. Koike K, Takaki A, Tatsukawa M, Suzuki M, Shiraha H, Iwasaki Y, Sakaguchi K and Shiratori Y: Combination of 5-FU and IFNalpha enhances IFN signaling pathway and caspase-8 activity, resulting in marked apoptosis in hepatoma cell lines. Int J Oncol 29: 1253-1261, 2006.

5. Wu XQ, Dai Y, Yang Y, Huang C, Meng XM, Wu BM and Li J: Emerging role of miRNAs in regulating macrophage activation and polarization in immune response and inflammation. Immunology 148: 237-248, 2016.

6. Piontek K and Selaru FM: MicroRNAs in the biology and diagnosis of cholangiocarcinoma. Semin Liver Dis 35: 55-62, 2015.

7. Livak KJ and Schmittgen TD: Analysis of relative gene expression data using real-time quantitative PCR and the 2(-Delta Delta C(T)) method. Methods 25: 402-408, 2001.

8. Ge YS, Xu GL, Zhang CH, Jia WD, Li JS, Ma JL and Yu JH: Efficacy and feasibility of radiofrequency ablation for hepatocellular carcinoma patients. Hepatogastroenterology 59: 2540-2542, 2012.

9. Safran H, Charpentier KP, Kaubisch A, Mantripragada K, Dubel G, Perez K, Faricy-Anderson K, Miner T, Eng Y, Victor $\mathrm{J}$, et al: Lenalidomide for second-line treatment of advanced hepatocellular cancer: A brown university oncology group phase II study. Am J Clin Oncol 38: 1-4, 2015.

10. Zhu AX, Holalkere NS, Muzikansky A, Horgan K and Sahani DV: Early antiangiogenic activity of bevacizumab evaluated by computed tomography perfusion scan in patients with advanced hepatocellular carcinoma. Oncologist 13: 120-125, 2008.

11. Saxena NK, Sharma D, Ding X, Lin S, Marra F, Merlin D and Anania FA: Concomitant activation of the JAK/STAT, $\mathrm{PI} 3 \mathrm{~K} / \mathrm{AKT}$, and ERK signaling is involved in leptin-mediated promotion of invasion and migration of hepatocellular carcinoma cells. Cancer Res 67: 2497-2507, 2007.
12. Lejeune D, Dumoutier L, Constantinescu S, Kruijer W, Schuringa JJ and Renauld JC: Interleukin-22 (IL-22) activates the JAK/STAT, ERK, JNK, and p38 MAP kinase pathways in a rat hepatoma cell line. Pathways that are shared with and distinct from IL-10. J Biol Chem 277: 33676-33682, 2002.

13. Schaper F, Siewert E, Gomez-Lechon MJ, Gatsios P, Sachs M, Birchmeier W, Heinrich PC and Castell J: Hepatocyte growth factor/scatter factor (HGF/SF) signals via the STAT3/APRF transcription factor in human hepatoma cells and hepatocytes. FEBS Lett 405: 99-103, 1997.

14. Inamura K, Matsuzaki Y, Uematsu N, Honda A, Tanaka N and Uchida K: Rapid inhibition of MAPK signaling and anti-proliferation effect via JAK/STAT signaling by interferon-alpha in hepatocellular carcinoma cell lines. Biochim Biophys Acta 1745: 401-410, 2005.

15. Kang FB, Wang L, Jia HC, Li D, Li HJ, Zhang YG and Sun DX: B7-H3 promotes aggression and invasion of hepatocellular carcinoma by targeting epithelial-to-mesenchymal transition via JAK2/STAT3/Slug signaling pathway. Cancer Cell Int 15: 45, 2015.

16. Hunecke D, Spanel R, Langer F, Nam SW and Borlak J: MYC-regulated genes involved in liver cell dysplasia identified in a transgenic model of liver cancer. J Pathol 228: 520-533, 2012.

17. Liu H, Radisky DC, Yang D, Xu R, Radisky ES, Bissell MJ and Bishop JM: MYC suppresses cancer metastasis by direct transcriptional silencing of alphav and beta3 integrin subunits. Nat Cell Biol 14: 567-574, 2012.

18. Li L, Jin R, Zhang X, Lv F, Liu L, Liu D, Liu K, Li N and Chen D: Oncogenic activation of glypican-3 by c-Myc in human hepatocellular carcinoma. Hepatology 56: 1380-1390, 2012.

19. Leskov I, Pallasch CP, Drake A, Iliopoulou BP, Souza A, Shen CH, Schweighofer CD, Abruzzo L, Frenzel LP, Wendtner CM, et al: Rapid generation of human B-cell lymphomas via combined expression of $\mathrm{Myc}$ and $\mathrm{Bcl} 2$ and their use as a preclinical model for biological therapies. Oncogene 32: 1066-1072, 2013.

20. Zhang XW and Xu B: Differential regulation of P53, c-Myc, $\mathrm{Bcl}-2$, Bax and AFP protein expression, and caspase activity during 10-hydroxycamptothecin-induced apoptosis in Hep G2 cells. Anticancer Drugs 11: 747-756, 2000.

21. Wang Z, Medrzycki M, Bunting ST and Bunting KD: Stat5-deficient hematopoiesis is permissive for Myc-induced B-cell leukemogenesis. Oncotarget 6: 28961-28972, 2015. 